\title{
Philosophiques
}

Donald Peterson, Wittgenstein's Early Philosophy. Three Sides of the Mirror, Toronto/Buffalo, University of Toronto Press, 1990, 204 pages. ISBN: 0-8020-2770-9.

\section{André Leclerc}

Volume 18, numéro 1, printemps 1991

URI : https://id.erudit.org/iderudit/027146ar

DOI : https://doi.org/10.7202/027146ar

Aller au sommaire du numéro

Éditeur(s)

Société de philosophie du Québec

ISSN

0316-2923 (imprimé)

1492-1391 (numérique)

Découvrir la revue

Citer ce compte rendu

Leclerc, A. (1991). Compte rendu de [Donald Peterson, Wittgenstein's Early Philosophy. Three Sides of the Mirror, Toronto/Buffalo, University of Toronto Press, 1990, 204 pages. ISBN: 0-8020-2770-9.] Philosophiques, 18(1), 179-182.

https://doi.org/10.7202/027146ar d'utilisation que vous pouvez consulter en ligne.

https://apropos.erudit.org/fr/usagers/politique-dutilisation/ 
DONALD PETERSON, Witgenstedn's Early Pbilosopby. Three Sides of the Mirror, Toronto/Buffalo, University of Toronto Press, 1990, 204 pages. ISBN : 0-8020-2770-9.

\author{
par André Leclerc
}

Écrire un commentaire - un autre - sur le Tractatus de Wittgenstein représente un défi de taille. Peu d'ouvrages ont exercé une fascination comparable sur les philosophes contemporains. Que d'éloges, de critiques, et d'interprétations en moins d'un siècle! Ouvrir à nouveau le dossier, le soumettre à un nouvel examen et proposer une interprétation originale et intéressante sans retomber constamment dans les redites, c'est en soi un bel accomplissement dont notre auteur peut certes être fier.

Le Tractatus de Wittgenstein contient, comme chacun sait, des considérations philosophiques d'une étonnante variété, et il fallait sans doute le génie de Wittgenstein pour les faire entrer toutes dans une seule et même perspective. Mais le Tractatus fait moins de cent pages dans la plupart des éditions et plusieurs de ces aphorismes ont un caractère sibyllin qui masque souvent l'unité de l'ouvrage. La principale thèse de Peterson est que les obscurités du Tractatus proviennent de ce que le plan d'ensemble ou le schème conceptuel (conceptual scbeme) de l'ouvrage n'est jamais rendu explicite. Ce schème conceptuel fournit la matrice à l'intérieur de laquelle sont abordées les questions relatives à l'ontologie, au langage représentationnel, au statut de la logique, au discours scientifique, à l'éthique, à la mystique et à la nature de la philosophie. Une fois dégagé, ce schème, que l'auteur désigne métaphoriquement comme les * trois faces du miroir *, jette un éclairage nouveau sur les diverses parties de l'ouvrage et sur sa progression.

Le Tractatus est avant tout un traité de philosophie du langage, et Wittgenstein compare lui-même le langage à un *grand miroir» (cf. 5.511). Mais contrairement à beaucoup d'autres traités sur le même sujet, le Tractatus s'occupe des trois côtés du miroir :

$\left.1^{\circ}\right)$ la réflexion ou représentation du monde des faits dans le langage - l'ontologie et la structure des énoncés élémentaires du langage représentationnel; $2^{\circ}$ ) la structure et les caractéristiques internes du miroir - la logique et le domaine de la syntaxe; et $3^{\circ}$ ) l'envers du miroir, ce qui se trouve derrière lui et ne peut s'y réfléchir - l'ineffable et le domaine de la mystique. Nous avons ainsi trois domaines et trois types d'énoncé correspondants.

D'abord le domaine des * faits * (Tatsachen), dont la totalité constitue le * monde * et qui s'analysent en * faits élémentaires * (Sacbverbalte). Les faits sont tout ce qui peut être dit ; ils sont décrits par les énoncés du langage représentationnel (les seuls qui aient un sens), et principalement par ceux des sciences de la nature.

Ensuite, celui de la logique et de la syntaxe (the syntactic) comprenant les propriétés et les relations internes des énoncés du langage représentationnel. Ces propriétés et relations internes peuvent être montrées mais non pas dites. Wittgenstein s'oppose constamment à la tendance qu'on a trop souvent eue de concevoir ces propriétés et relations internes comme appartenant au monde plutôt qu'au langage. 
En conséquence, les énoncés de la logique sont dépourvus de sens (sinnloss), non parce qu'ils seraient incompréhensibles, mais plutôt parce que le langage est alors utilisé d'une manière réflexive ou non représentationnelle pour parler des énoncés du langage représentationnel, et non à propos des faits ( La logique est transcendantale *, $c f, 6.13$ ). Il y a enfin le domaine de l'ineffable (the mystical), et les * pseudo-énoncés * qui s'y rapportent sont « insensés * (unsinnig).

Dans la Préface du Tractatus, Wittgenstein paraît plutôt indiquer un schème conceptuel plus simple, son but étant de tracer une limite à l'expression des pensées; ce qui se trouve de l'autre côté de cette limite ne sera donc que non-sens. Mais cette division plus simple en sens et non-sens ne rend manifestement pas justice à la complexité de l'ouvrage. Le schème proposé par Peterson rend mieux compte de la progression de l'ouvrage et de la distance que met Wittgenstein lui-même entre les énoncés de la logique (sinnloss), qui sont des tautologies ( $c f, 6.112$, 6.1), et ceux de la mystique (unsinnig), des "pseudo-énoncés" qui prétendent dire quelque chose là où rien ne peut être dit.

Peterson n'affirme pas que le schème conceptuel qu'il dégage du Tractatus parvient à intégrer sans problème tous les thèmes philosophiques abordés dans l'opuscule de Wittgenstein. Au contraire, à tout moment Peterson, en critique perspicace et jamais à court d'arguments, fait voir un grand nombre de difficultés soulevées par la perspective du Tractatus. Ainsi, Wittgenstein n'arrive pas vraiment à tracer la limite du dicible; $d$ 'abord parce que le Tractatus ne fournit aucune méthode d'analyse permettant d'identifier ses a configurations d'objets" (faits ou états de choses) et que pour décider si quelque chose est d'ordre factuel ou non, il faudrait pouvoir décider si nous avons affaire ou non à un fait (ou état de choses); ensuite parce que le Tractatus ne dit pas non plus quand un énoncé du langage ordinaire a un sens ou quelle méthode on doit suivre pour le réduire à une fonction de vérité d'énoncés élémentaires. Les métaphores et le discours poétique peuvent-ils constituer d'authentiques descriptions de faits ou d'états de choses ? Il est évident que le langage des mystiques, des moralistes, ou encore les descriptions de nos expériences esthétiques, sont truffés de métaphores. On peut soutenir que non, mais il faut reconnaître que dans le langage ordinaire en constante évolution, les descriptions littérales d'aujourd'hui sont souvent les métaphores d'hier. En l'absence de critères permettant d'identifier ce qui compte comme fait (ou état de choses) ou d'identifier les énoncés du langage ordinaire qui sont d'authentiques descriptions, la limite du dicible que Wittgenstein se proposait de tracer est donc inopérante ou inutilisable. Toutefois, en dépit des lacunes sur des points de détail, ce qui fait la valeur de l'héritage du Tractatus selon Peterson, ce n'est pas l'une ou l'autre de ses doctrines particulières, qui sont relativement indépendantes les unes des autres, mais la perspective qui les intègre, les "trois faces du miroir" (the three-sided mirror). Son principal mérite et son originalité lui viennent de ce qu'il montre que les trois faces du miroir ne sont pas incommensurables et peuvent être considérées simultanément.

Il y a un autre point fort du commentaire de Peterson que $\mathrm{j}$ 'aimerais souligner. Aucun autre commentaire, à ma connaissance, ne fait voir aussi clairement toutes les implications de la fameuse Grundgedanke de 
Wittgenstein, la thèse voulant que les constantes logiques ne représentent pas. Wittgenstein ne l'appelle pas sa * pensée fondamentale * pour rien. Elle fonde la théorie des fonctions de vérité, complète la théorie de la * proposition-image * (picture tbeory of meaning), conduit à la thèse de l'indépendance logique des énoncés élémentaires, détermine la conception des propositions de la logique en tant que tautologies, et celles de la logique comme étant purement * syntaxiques *. La Grundgedanke informe également la philosophie des mathématiques et des sciences de Wittgenstein. La mise au jour de toutes les implications de cette thèse fait voir clairement la remarquable unité qui règne dans le Tractatus.

Peterson termine son ouvrage sur deux épilogues et un appendice. Le premier épilogue, intitulé * The Tractatus and Cognitive Science*, discute très brièvement de certaines idées du Tractatus en relation avec les sciences cognitives (la spécialité de l'auteur), et procède entre autres à une intéressante comparaison entre la théorie de la proposition-image et la théorie du langage mental de Fodor. Mais cette discussion, pour intéressante qu'elle soit, est trop brève (à peine cinq pages) pour combler les attentes du lecteur (la jaquette du livre donne à penser que l'auteur appliquera les idées de Wittgenstein sur la logique et la représentation aux sciences cognitives, ce qui est loin d'être le cas). Le second épilogue, par contre, contient des suggestions fort intéressantes. Intitulé "The Tractatus and Abstract Art*, l'auteur y soutient que ce que le mouvement de l'art abstrait a fait pour la représentation visuelle, le Tractatus l'a fait pendant la même période pour la représentation linguistique. En renonçant à représenter le monde et ses objets, des peintres comme Kandinsky portent toute leur attention sur les lignes, les formes, les surfaces et les couleurs, dont les propriétés et relations sont internes à la représentation picturale. De même, la logique, pour Wittgenstein, s'occupe de ce qui est présent dans nos représentations linguistiques, et non de ce qui est représenté par elles. La logique selon Wittgenstein et l'art abstrait affirment, chacun à leur manière, leur autonomie : ils peuvent "prendre soin d'eux-mêmes "...

L'appendice concerne les difficultés liées à la traduction des termes Tatsacbe, Sachverhalt, Satz et Elementarsatz, et rejette en particulier la thèse de Stenius voulant que les Sachverbalte soient des faits possibles. Il s'agit plutôt, selon Peterson, de faits actuels ontologiquement simples dont se composent les Tatsacben. L'argument, trop long pour être ici rapporté, est élégant et bien mené.

Il s'agit donc en résumé d'un commentaire intéressant, proposant une interprétation originale construite autour de l'hypothèse d'un schème conceptuel informant le Tractatus tout entier. La métaphore des trois côtés du miroir ne rend peut-être pas justice à l'idée que l'auteur se fait du schème en question, mais elle est suggestive. L'auteur fait bien quelques remarques ici et là sur les rapports entre les « deux philosophies ※ de Wittgenstein, mais il ne fait nulle part le point sur ce qui subsiste du schème ou de la perspective du Tractatus dans la seconde philosophie, ou s'il pourrait servir à expliquer le passage de l'une à l'autre. L'ouvrage est bien documenté, fait le point sur plusieurs controverses, et s'il ne dit malheureusement rien sur certains aphorismes d'un caractère toujours obscur (comme ceux concernant la 
psychologie ou l'interprétation des énoncés de la forme *A croit que $P$. - cf. 5.542 et suivants), il permet à coup sûr un approfondissement des thèmes philosophiques majeurs du Tractatus.

Université du Québec

a Trois-Rivières 